\title{
\begin{tabular}{l|l} 
Mibraries & DSpace@MIT
\end{tabular}
}

\author{
MIT Open Access Articles
}

\section{Self-Stratification of Tropical Cyclone Outflow. Part II: Implications for Storm Intensification}

The MIT Faculty has made this article openly available. Please share how this access benefits you. Your story matters.

Citation: Emanuel, Kerry. “Self-Stratification of Tropical Cyclone Outflow. Part II: Implications for Storm Intensification." Journal of the Atmospheric Sciences 69.3 (2012): 988-996. (C) 2012 American Meteorological Society

As Published: http://dx.doi.org/10.1175/jas-d-11-0177.1

Publisher: American Meteorological Society

Persistent URL: http://hdl.handle.net/1721.1/73666

Version: Final published version: final published article, as it appeared in a journal, conference proceedings, or other formally published context

Terms of Use: Article is made available in accordance with the publisher's policy and may be subject to US copyright law. Please refer to the publisher's site for terms of use. 


\title{
Self-Stratification of Tropical Cyclone Outflow. Part II: Implications for Storm Intensification
}

\author{
KERRY EMANUEL \\ Program in Atmospheres, Oceans, and Climate, Massachusetts Institute of Technology, Cambridge, Massachusetts
}

(Manuscript received 1 July 2011, in final form 26 October 2011)

\begin{abstract}
Tropical cyclones intensify and are maintained by surface enthalpy fluxes that result from the thermodynamics disequilibrium that exists between the tropical oceans and atmosphere. While this general result has been known for at least a half century, the detailed nature of feedbacks between thermodynamic and dynamic processes in tropical cyclones remains poorly understood. In particular, the spatial relationship between surface fluxes and the radial entropy distribution apparently does not act to amplify the entropy gradient and therefore the surface winds. In previous work, this problem was addressed by accounting for the radial distribution of convective fluxes of entropy out of the boundary layer; this led to the conclusion that a radial gradient of such convective fluxes is necessary for intensification.

Part I showed that the assumption of constant outflow temperature is incorrect and argued that the thermal stratification of the outflow is set by small-scale turbulence that limits the Richardson number. The assumption of Richardson number criticality of the outflow allows one to derive an equation for the variation of outflow temperature with angular momentum; this in turn leads to predictions of vortex structure and intensity that agree well with tropical cyclones simulated using a full-physics axisymmetric model. Here it is shown that the variation of outflow temperature with angular momentum also permits the vortex to intensify with time even in the absence of radial gradients of entrainment into the boundary layer. An equation is derived for the rate of intensity change and compared to simple models and to simulations using a full-physics model.
\end{abstract}

\section{Introduction}

Tropical cyclones are a form of natural heat engine that absorb enthalpy from the ocean at the relatively high temperatures of the tropical sea surface and eject it at the very low temperatures of the tropical tropopause layer (Riehl 1950; Emanuel 1986). Large rates of enthalpy transfer from the sea surface are made possible by the thermodynamic disequilibrium that exists between the tropical oceans and atmosphere, a consequence of the greenhouse effect (Emanuel 2007). The rapid dissipation of tropical cyclones once they move over land testifies to the essential role of local sea-air enthalpy transfer (as opposed to ambient convective available potential energy) in maintaining the storms.

While the overall energetics of tropical cyclones are reasonably well understood, the details of the processes

Corresponding author address: Kerry Emanuel, Rm. 54-1814, Massachusetts Institute of Technology, 77 Massachusetts Avenue, Cambridge, MA 02139.

E-mail: emanuel@mit.edu by which existing storms intensify are less so. The author (Emanuel 1997, hereafter E97) attempted an examination of these processes using a highly simplified model in which the vortex is considered to be nearly in hydrostatic and gradient wind balance at all times, in a state of neutrality to slantwise moist convection, and bounded at the top by a well-developed anticyclone. This work showed that the eyewall region is highly frontogenetic and that without nonaxisymmetric turbulence, frontal collapse would take place and limit further intensification of the storm. Another significant-but disturbingfinding of E97 was that the phase relationship between surface enthalpy fluxes and the existing radial distribution of boundary layer entropy does not, by itself, allow for the intensification of the vortex. In essence, the radial distribution of surface enthalpy fluxes does not act to increase the magnitude of the radial gradient of entropy, a direct measure of the intensity of the storm. E97 argued that a plausible radial gradient of convective entropy fluxes out of the boundary layer is needed to allow intensification. He introduced a factor $\beta$ multiplying the local entropy tendency and showed that 
a radial gradient of $\beta$ across the eyewall region is necessary for intensification. The lack of an explicit closure for convective entropy fluxes out of the boundary layer is a decided limitation of that work.

A common assumption used in constructing the balanced, convectively neutral vortices used in E97 and other works is that all the streamlines emanating from the boundary layer asymptote to the undisturbed environmental entropy surfaces matching the saturation entropy of the streamlines, and that these surfaces can be assumed to lie in the lower stratosphere at approximately constant absolute temperature. Recently, Emanuel and Rotunno (2011, hereafter Part I) demonstrated that in numerically simulated tropical cyclones, the assumption of constant outflow temperature is poor and that, in the simulations, the outflow temperature increases rapidly with angular momentum. They postulated that the absolute temperature stratification of the outflow is determined by small-scale turbulence that limits the Richardson number to a critical value for the onset of turbulence, and showed that such an assumption leads to an equation for the outflow temperature as a function of absolute angular momentum $M$ :

$$
\frac{\partial T_{o}}{\partial M} \cong-\frac{\mathrm{Ri}_{c}}{r_{t}^{2}}\left(\frac{d M}{d s^{*}}\right)
$$

where $T_{o}$ is the outflow temperature, $s^{*}$ is the saturation entropy, $\mathrm{Ri}_{c}$ is the critical Richardson number, and $r_{t}$ is the physical radius at which the Richardson number first attains its critical value. Based mostly on analysis of numerical simulations using the model of Rotunno and Emanuel (1987, hereafter RE87), Part I applied to (1) the boundary condition that $T_{o}$ is equal the ambient tropopause temperature $T_{t}$ for the particular streamline emanating from the boundary layer at the radius of maximum winds. When coupled with the thermal wind relation and equations for the evolution of entropy and angular momentum in the boundary layer, (1) yields solutions for the intensity and structure of mature, steadystate vortices that are in good accord with simulations using the RE87 model. ${ }^{1}$

We remark that the boundary condition applied to (1), $T_{o}=T_{t}$ at the angular momentum surface corresponding to the radius of maximum winds, has at present

\footnotetext{
${ }^{1}$ Note that in integrating (1), Part I held $r_{t}$ constant. We will make this assumption here as well, but note that the results may show some sensitivity to this assumption. A more thorough exploration of this assumption is left to future work. Note also that (1) is not expected to apply in the outer region of the vortex, where there is mean radial inflow and downflow. This issue is discussed more extensively in Part I.
}

no theoretical justification. We use it here because, empirically, it well describes the outflow temperature distribution in the numerical simulations reported in Part I (see, in particular, Fig. 5 therein). This boundary condition also corresponds to essentially no outflow into the ambient stratosphere where, owing to the high static stability present there, outflow would presumably need to do more work against the twin constraints of stratification and rotation. We leave for future work a more rigorous treatment of the important boundary condition need to solve (1).

The importance of the outflow temperature in the system can easily be seen in the structure of the thermal wind equation for a well-developed, balanced, convectively neutral vortex specialized to the case in which the vorticity is large compared to the Coriolis parameter [see (12) of Part I]:

$$
\frac{V_{b}}{r_{b}}=-\left(T_{b}-T_{o}\right) \frac{d s^{*}}{d M}
$$

where $V_{b}, r_{b}$, and $T_{b}$ are respectively the azimuthal velocity, physical radius, and absolute temperature evaluated at the top of the boundary layer. When this is coupled with equations governing the evolution of angular momentum and entropy in the boundary layer, an equation for the wind speed itself results (see Part I):

$$
V_{b}^{2} \cong \frac{C_{k}}{C_{D}}\left(T_{b}-T_{o}\right) \frac{\left(k_{0}^{*}-k\right)}{T_{s}},
$$

where $C_{k}$ and $C_{D}$ are the dimensionless exchange coefficients for enthalpy and momentum, $k_{0}^{*}$ and $k$ are the saturation enthalpy of the sea surface and actual enthalpy of the boundary layer, and $T_{s}$ is the sea surface temperature. With constant $T_{o}$, (3) would predict a highly unrealistic radial structure of the azimuthal velocity. But with $T_{o}$ determined using (1), quite reasonable radial profiles of $V_{b}$ are obtained. Moreover, the magnitude of the maximum value of $V_{b}$ (which depends, among other things, on the value of $k$ at the radius of maximum winds, which must be determined as part of the complete solution of the system) depends in realistic ways on environmental conditions and on the values of $C_{k}$ and $C_{D}$ (see Part I).

Our present purpose is to demonstrate that allowing the outflow temperature to vary according to (1) also results in a more realistic picture of the intensification of tropical cyclones and allows one to dispense with the $\beta$ factor introduced by E97. The next section derives equations for the intensification of tropical cyclones starting from well-developed vortices, parallel to E97 but allowing for variable outflow temperature. These 
equations are then specialized in section 3 to the inner core region and an approximate analytic formula for the time evolution of the maximum wind speed is derived. Section 4 presents numerical solutions of the full equations and compares them to the simple analytic equation for the maximum wind speed. Comparisons to simulations using the RE87 model will also be made. These results will be discussed and summarized in section 5 .

\section{Time-dependent system}

Following E97, we assume that the time scale of tropical cyclone intensification is long enough that the vortex (above the boundary layer) can be considered to evolve through a sequence of quasi-balanced states for which the thermal wind equation, based on hydrostatic and gradient wind balance, always applies. The complete form of the thermal wind equation [omitting the assumption of large vorticity used in deriving (2)] can be written (cf. Emanuel 1986)

$$
\frac{M}{r_{b}^{2}}=\frac{M}{r_{o}^{2}}-\left(T_{b}-T_{o}\right) \frac{d s^{*}}{d M},
$$

where $r_{o}$ is the physical radius of an angular momentum surface at the point where the absolute temperature is $T_{o}$. Following Part I, we define that point as the point along the angular momentum surface at which the azimuthal velocity changes sign. At that point, by definition, $M=(1 / 2) f r_{o}^{2}$ and so we can write (4) in the form of an equation for $r_{b}^{2}$ :

$$
r_{b}^{2}=\frac{M}{(1 / 2) f-\left(T_{b}-T_{o}\right)\left(d s^{*} / d M\right)} .
$$

Given $r_{b}$ from (5), the azimuthal velocity may be found from the definition of $M$ [i.e., $V=M / r-(1 / 2) f r$ ]. To close the system, we need to determine $s^{*}$ and $T_{o}$ as functions of time and $M$. To do this we separate the radial structure of the vortex into two distinct regions: the eye and the rest of the system. Following one of the three cases examined in E97, we assume that the eye is in solid body rotation and determine the distribution of saturation entropy there using the high-vorticity approximation to (4) given by (2). In the rest of the vortex, we assume, as did E97, that the saturation entropy in the free atmosphere (along angular momentum surfaces) equals the actual entropy of the boundary layer $s_{b}$. The latter is determined through an evolution equation written in angular momentum coordinates (see Part I):

$$
\frac{\partial s_{b}}{\partial \tau}+\dot{M} \frac{\partial s_{b}}{\partial M}=g \frac{\partial F}{\partial P}+D,
$$

where $\dot{M}$ is the total time derivative of angular momentum, $F$ is the vertical turbulent flux of entropy, and $D$ represents the irreversible entropy sources owing to kinetic energy dissipation, nonequilibrium evaporation of liquid water, and diffusion of water vapor. The angular momentum source may be derived from the azimuthal momentum equation:

$$
\dot{M}=g r \frac{\partial \tau_{\theta}}{\partial P},
$$

where $\tau_{\theta}$ is the tangential stress and we have neglected advection of entropy along angular momentum surfaces in the boundary layer. Substituting (7) into (6) and integrating over the pressure depth of the boundary layer $\Delta p_{b}$ yields

$$
\Delta p_{b} \frac{\partial s_{b}}{\partial \tau}+g r \tau_{\theta s} \frac{\partial s_{b}}{\partial M}=g F_{s}+\bar{D},
$$

where $\tau_{\theta s}$ is the azimuthal surface stress, $F_{s}$ is the surface enthalpy flux divided by the surface temperature, and $\bar{D}$ represents the irreversible entropy sources integrated in pressure over the boundary layer. Note that, in contrast to E97, we omit any turbulent entropy fluxes out of the top of the boundary layer; nor do we include any effects of vertical advection into the boundary layer. We do this deliberately to focus on the role of varying outflow temperature. Likewise, E97 showed that varying boundary layer depth could yield intensification. Our boundary layer depth could be interpreted as the "thermodynamic boundary layer depth" in the terminology of Zhang et al. (2011), who showed from detailed boundary layer observations of hurricanes that this boundary layer depth generally increases with radius in hurricanes, although probably not fast enough to yield intensification according to the E97 model. Here again we ignore this effect of varying boundary layer depth so as to focus on the effects of variable outflow temperature, noting that in any case variable $\Delta p_{b}$ cannot contribute to the steady-state structure as it multiplies a local time derivative.

We use the neutral aerodynamic flux formulas for $\tau_{\theta s}$ and $F_{s}$ :

$$
F_{s}=\frac{C_{k} \rho|\mathbf{V}|\left(k_{0}^{*}-k_{b}\right)}{T_{s}} \cong C_{k} \rho|\mathbf{V}|\left(s_{0}^{*}-s_{b}\right)
$$

and

$$
\tau_{\theta s}=-C_{D} \rho|\mathbf{V}| V
$$

where $\rho$ is the surface air density, $|\mathbf{V}|$ is the wind speed, $k_{b}$ is the actual enthalpy of air at the reference level at which the wind speed and exchange coefficients are 
defined, and the other terms are defined above. In (9) we introduce the reasonable approximation that $k_{0}^{*}-k_{b} \cong$ $T_{s}\left(s_{0}^{*}-s_{b}\right)$, where $s_{0}^{*}$ is the saturation entropy of air at sea surface temperature and pressure. ${ }^{2}$ Following Bister and Emanuel (1998), we represent the vertically integrated dissipative heating as

$$
\bar{D} \cong g \rho \frac{C_{D}|\mathbf{V}|^{3}}{T_{s}} .
$$

Substituting (9)-(11) into (8) yields

$$
h \frac{\partial s_{b}}{\partial \tau}-C_{D} r|\mathbf{V}| V \frac{\partial s_{b}}{\partial M}=C_{k}|\mathbf{V}|\left(s_{0}^{*}-s_{b}\right)+C_{D} \frac{|\mathbf{V}|^{3}}{T_{s}}
$$

where $h$ is a boundary layer depth scale defined as

$$
h \equiv \frac{\Delta p_{b}}{\rho g} .
$$

In this simple system, the entire time dependence is contained in (12), the evolution equation for boundary layer entropy. Except in the eye, we are assuming that $s^{*}=s_{b}$, a condition for slantwise convective neutrality. We define the eye as the region inside the radius of maximum winds and assume solid body rotation there. We also assume that the outflow temperature is equal to the ambient tropopause temperature everywhere at and inside the radius of maximum winds, following Part I. We then use (2) together with the assumption of solid body rotation to calculate the saturation entropy everywhere inside the radius of maximum winds, using as a boundary condition $s^{*}=s_{b}$ at the radius of maximum winds. Note that we solve (12) everywhere, including in the eye, though in this case the boundary layer entropy is decoupled from (and is less than) the saturation entropy above the boundary layer.

We now have a closed system consisting of (12), (5), and (1) together with $V=M / r-(1 / 2) f r$, the boundary condition that $T_{o}=T_{t}$ at and inside the radius of maximum winds, and the condition of solid body rotation inside the radius of maximum winds. As demonstrated by E97, imposing this solid body rotation in the eye (equivalent to having large horizontal turbulent mixing) prevents formation of a discontinuity resulting from eyewall frontogenesis. We also apply $s^{*}=s_{b}$, except in the eye, and following Part I take $r_{t}$ in (1) to be a constant.

\footnotetext{
${ }^{2}$ The effects of irreversible evaporation and diffusion of water vapor could be accounted for by replacing $T_{s}$ in this equation by $T_{b}$, the temperature at the top of the subcloud layer, but we neglect this small difference here.
}

In some ways this system is analogous to the classical quasigeostrophic Eady model. As in the Eady case, an invertible, potential vorticity-like quantity, the saturation potential vorticity, is assumed to be constant everywhere (and zero in this case, corresponding to slantwise convective neutrality). Also as in the Eady problem, all the time dependence enters through a conservation equation for entropy at the boundaries, although in this case a time-dependent equation obtains only for the lower boundary. The upper boundary condition is given by (1) and is diagnostic, not prognostic. Other important differences from the Eady problem are the circular geometry, the advection of entropy by the Ekman flow rather than by the balanced flow, and the essential importance of surface fluxes in the budget of boundary entropy.

In section 4 we will present numerical solutions to this system. Before we do so, we will examine an approximation to this system, valid in the inner core, and use it to argue for the essential importance of the radial variation of the outflow temperature given by (1) for the intensification of tropical cyclones.

\section{Approximate system}

Following E97, we derive an approximation to the system described in section 2 that is valid in the inner core region, where the relative vorticity is much larger than the Coriolis parameter. In that case, (5) reduces to the approximate form (2) and we can assume that $M \simeq$ $r V$. In addition to this, we apply further approximations:

(a) neglect the pressure dependence of $s_{0}^{*}$ that appears in (12);

(b) neglect dissipative heating, the last term in (12);

(c) approximate both $|\mathbf{V}|$ and $V$ in (12) by the gradient wind at the top of the boundary layer, which for simplicity we will simply denote as $V$; and

(d) approximate the thermodynamic boundary layer depth $h$ as a constant.

As we apply these approximations in the eyewall region, we assume that $s_{b}=s^{*}$. Then our approximate system is

$$
\begin{gathered}
V^{2}=-\left(T_{b}-T_{o}\right) M \frac{\partial s^{*}}{\partial M}, \\
\frac{\partial T_{o}}{\partial M}=-\frac{\operatorname{Ri}_{c}}{r_{t}^{2}}\left(\frac{\partial s^{*}}{\partial M}\right)^{-1}, \\
h \frac{\partial s^{*}}{\partial \tau}-C_{D} V M \frac{\partial s^{*}}{\partial M}=C_{k} V\left(s_{0}-s^{*}\right),
\end{gathered}
$$

where $s_{0}$ is the environmental (constant) saturation entropy of air at sea surface temperature and ambient surface pressure. 
While the system (13)-(15) does not appear to have an analytic solution, certain features of the behavior of the solutions can be exposed by first differentiating (15) with respect to $M$, using (13) to eliminate $\partial s^{*} / \partial M$, and using (14) for $\partial T_{o} / \partial M$. The result is

$$
\begin{aligned}
\frac{\left(T_{b}-T_{o}\right) h}{V} \frac{\partial}{\partial \tau}\left(\frac{V^{2}}{T_{b}-T_{o}}\right)= & \frac{M}{V} \frac{\partial V}{\partial M}\left[3 C_{D} V^{2}\right. \\
& \left.-C_{k}\left(T_{b}-T_{o}\right)\left(s_{0}-s^{*}\right)\right] \\
& +C_{D} \frac{\mathrm{Ri}_{c}}{r_{t}^{2}} M^{2}-C_{k} V^{2}
\end{aligned}
$$

This is not a closed expression owing to the appearance of $s^{*}$ in the first term on the right as well as to the dependence of $T_{o}$ on $s^{*}$ through (14). The important conclusion to be drawn from (16) is that at the radius of maximum wind, where $T_{o}=T_{t}$ and $\partial V / \partial M=0$, the time tendency of azimuthal wind would be negative were it not for the second term on the right-hand side of (16), which arises [through (14)] from the radial gradient of outflow temperature. Thus, in this formulation the amplification of the vortex depends on the radial gradient of outflow temperature, which in turn, according to the results of Part I, is a result of small-scale turbulence in the outflow region.

A casual glance at (16) would appear to suggest that the amplification rate varies positively with the drag coefficient but negatively with the enthalpy exchange coefficient. But without solving the whole system we do not know in advance the angular momentum value $M_{\max }$ at which the maximum azimuthal velocity occurs. We do know, from Part I, what the steady-state solution to (16) is, including the maximum azimuthal velocity $V_{\text {max }}$. If we assume, provisionally, that $M_{\max }$ is constant in time (i.e., that the radius of maximum winds always lies on the same angular momentum surface) then $M_{\max } \simeq r_{\max } V_{\max }$, where $r_{\max }$ is the radius of maximum winds in the steady state. According to (43) of Part I, $r_{\text {max }}^{2}=r_{t}^{2}\left(C_{k} / C_{D}\right) \mathrm{Ri}_{c}^{-1}$; thus, at the radius of maximum winds, if the angular momentum there is constant, (16) becomes

$$
\frac{\partial V_{m}}{\partial \tau} \cong \frac{C_{k}}{2 h}\left(V_{\max }^{2}-V_{m}^{2}\right)
$$

where $V_{m}$ is the maximum value (over radius) of the azimuthal wind at any given time, and $V_{\max }$ is the steady-state maximum wind whose value (as a function of environmental and other physical parameters) is given in Part I:

$$
V_{\max }^{2}=\frac{C_{k}}{C_{D}}\left(\frac{1}{2} \frac{C_{k}}{C_{D}}\right)^{\left(C_{k} / C_{D}\right) /\left[2-\left(C_{k} / C_{D}\right)\right]}\left(T_{b}-T_{t}\right)\left(s_{0}-s_{e}^{*}\right),
$$

where $s_{e}^{*}$ is the value of $s^{*}$ in the undisturbed environment.

The solution of (17), given an initial condition $V=0$ at $\tau=0$, is

$$
V_{m}(\tau)=V_{\max } \tanh \left(\frac{C_{k} V_{\max }}{2 h} \tau\right) .
$$

If this is valid, then the rate of intensification depends primarily on $C_{k}$, with $C_{D}$ entering only insofar as it affects $V_{\max }$ through (18). As we will show in the next section, the numerical solutions of the full system conform reasonably well to (19).

\section{Numerical solutions and comparison with simulations using the RE87 model}

The system consisting of (1), (5), and (12), together with $V=M / r-(1 / 2) f r$ and the boundary condition $T_{o}=T_{t}$ at and inside the angular momentum value corresponding to the radius of maximum wind, is solved numerically using a simple procedure. The sole time-dependent equation (12) is stepped forward using a leapfrog scheme with an Asselin filter applied to prevent time splitting. This yields the boundary layer entropy as a function of angular momentum and time. The location $M_{\Omega}$ of the maximum value of $-d s / d M$ is noted; according to (2) this is also the location of maximum angular velocity $V / r$. [This will generally occur inside the radius of maximum winds, where $T_{o}$ is held fixed at the value $T_{t}$ that attains at the radius of maximum winds; thus we need not be concerned with maximizing $-\left(T_{b}-T_{o}\right) d s / d M$.] Outside $M_{\Omega}$ we assume that the saturation entropy above the boundary layer equals the boundary layer entropy. Inside $M_{\Omega}$ we assume solid body rotation, which from (2) is equivalent to assuming that $d s^{*} / d M$ is constant. We take its constant value to be the value of $d s / d M$ at $M_{\Omega}$, so that the angular velocity (but not the vorticity) is continuous across $M_{\Omega}$. Having thus determined the distribution of $s$ and $s^{*}$, we solve (1) by integrating outward from $M_{\Omega}$; this yields the distribution of $T_{o}$ outside of $M_{\Omega}$. (Inside $M_{\Omega}$, we assume that $T_{o}=T_{t}$, the ambient tropopause temperature.) Finally, we use (5) together with $V=M / r-$ (1/2) fr to diagnose the physical radius $r$ and $V$.

The entropy equation (12) entails a globally averaged source of entropy to the atmosphere. To reach a steady state, we add a constant sink of entropy meant to represent radiative cooling. ${ }^{3}$ To facilitate comparison to the

\footnotetext{
${ }^{3}$ Literally, radiative cooling of the boundary layer. But since the boundary layer entropy is tied to the tropospheric saturation entropy in regions of convection, this sink might equally well be regarded as operating in the free troposphere.
} 
simplified model developed in section 3, we omit the pressure dependence of the sea surface entropy in (12). Also, for numerical stability, we relax the outflow temperature toward the value given by integrating (1) rather than strictly enforcing (1). We use a relaxation time constant of 1 day for this purpose. The simulation is initialized with a weak warm-core vortex given by

$$
s^{*}=s_{i}^{*}-\left(s_{i}^{*}-s_{e}^{*}\right)\left[1-e^{-a\left(M / M_{o}\right)^{2}}\right],
$$

where $s_{i}^{*}$ is the maximum value of saturation entropy, achieved at the center of the vortex, $s_{e}^{*}$ is the saturation entropy of the unperturbed environment, $M_{o}$ is a reference value of angular momentum, and $a$ is a constant that determines the size of the initial vortex. Starting from this initial condition, the system is integrated until an approximate steady state is achieved. Solutions are not sensitive to the initial intensity of the vortex as represented here by $s_{i}^{*}-s_{e}^{*}$; nor are they sensitive to the value of $a$ unless it is too small, corresponding to too large an initial vortex (as discussed, for example, in RE87).

It should be remarked that through a suitable normalization of the dependent and independent variables of this system, it can be shown that, aside from parameters related to radiative cooling and the initial condition, the nondimensional system behavior is governed by only two nondimensional parameters. If we define a velocity scale $V_{p}$ such that

$$
V_{p}^{2} \equiv\left(T_{b}-T_{t}\right)\left(s_{0}-s_{e}^{*}\right),
$$

and scale all length scales by $V_{p} / f$ and time by $h / C_{k} V_{p}$, the two governing nondimensional parameters are

$$
C_{r} \equiv \frac{C_{k}}{C_{D}} \quad \text { and } \quad \alpha \equiv \frac{\mathrm{Ri}_{c} V_{p}^{2}}{r_{t}^{2} f^{2}}
$$

As shown in Part I, $C_{r}$ affects both the intensity and the shape of the vortex, while $\alpha$ is a size parameter affecting the dimensions of the vortex. According to Part I, there is a well-defined steady-state solution that depends on $C_{r}$ and $\alpha$ but not the initial condition (except insofar is it affects $r_{t}$ ).

Bearing in mind that the fundamental nature of the solutions depends on the nondimensional parameters given by (21), we nonetheless present solutions in dimensional form for ease of interpretation. Experiments show that the intensity evolution is insensitive to $\alpha$ as long as it is sufficiently large, corresponding to an outer vortex radius much smaller than $V_{p} / f$. This is broadly

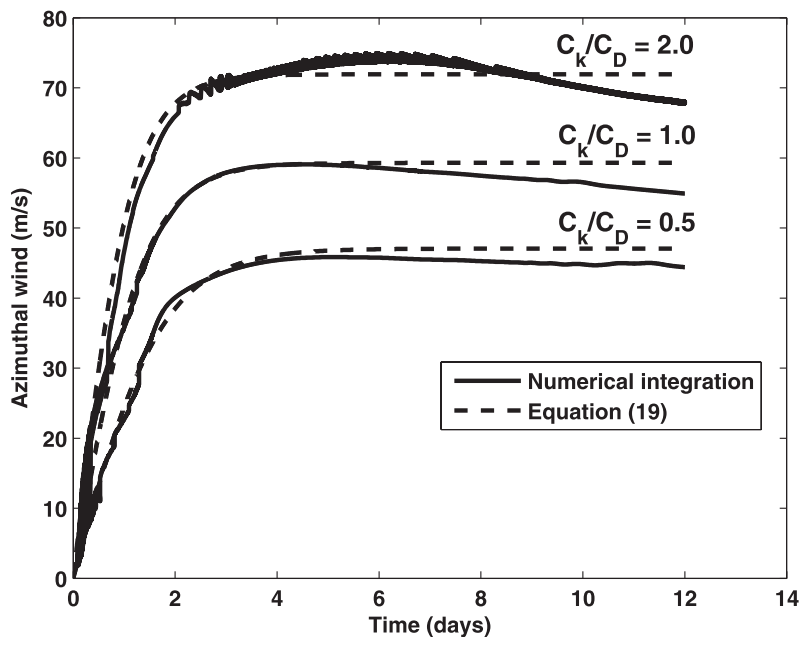

FIG. 1. Evolution with time of the peak azimuthal wind in the numerical simulations (solid) compared to the analytic approximation (19) (dashed) for three values of the ratio of exchange coefficients and with $\alpha=500$. In these simulations, the drag coefficient has been varied to control the variation of the ratio of exchange coefficients.

consistent with the findings of RE87, who showed that the nature of the simulations is not terribly sensitive to vortex size as long as it is not too large. In the results presented here, we set $\alpha=500$.

Figure 1 compares the time evolution of the maximum wind speed to the analytic approximation (19) for three values of $C_{r}$. For the purposes of this figure, $C_{r}$ has been varied by varying $C_{D}$ so as to keep the dimensional time on the same scale. Again for dimensional display purposes, we set $V_{p}$ to $84 \mathrm{~m} \mathrm{~s}^{-1}, f=5 \times 10^{-5} \mathrm{~s}^{-1}$, and $h=5000 \mathrm{~m}$.

Note that in view of the approximations underlying (19) and the addition of radiative cooling and temperature relaxation to the numerical simulation, there is no reason to expect the numerical solutions to be identical to (19). Nonetheless, (19) clearly captures most of the characteristics of the time evolution of the simulated azimuthal wind. (Note that if the simulations are extended in time, a true steady state is achieved.)

Figure 2 compares the steady-state solution using $C_{r}=1$ to that obtained in Part I, where a good analytic approximation to the steady solution of (1), (5), and (12) is developed. The small differences may be owing to the constant cooling applied to the time-dependent model. The profile in the outer region drops off too sharply compared to storms numerically simulated using the RE87 model. This is likely a result of the fact that (1) cannot be expected to apply in the outer region (see Part I), and the circumstance that the RE87 model uses Newtonian damping of temperature back to the initial state, thereby underestimating radiative cooling in the outer region (Hakim 2011). Since Ekman suction must 


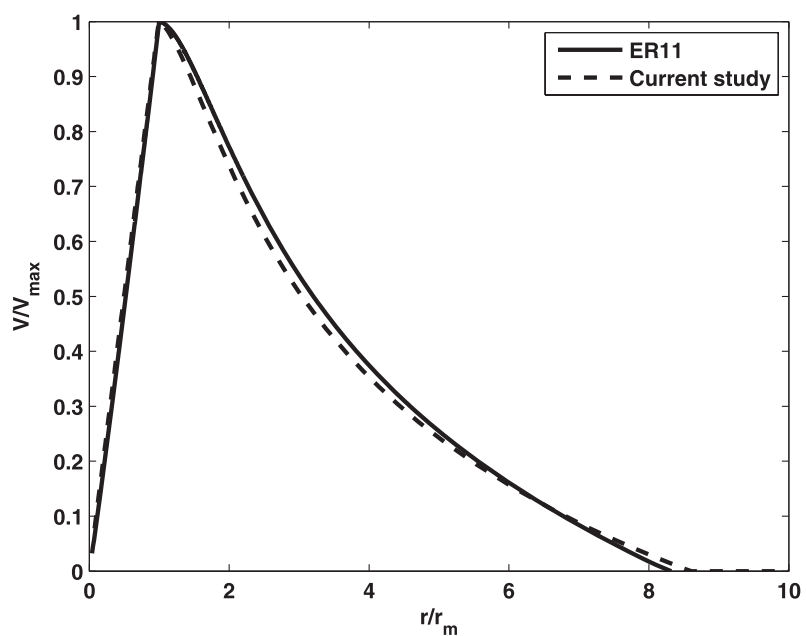

FIG. 2. Radial profile of azimuthal velocity from the steady-state model of Part I (solid) and at day 12 in the simulation using the time-dependent model (dashed); in both cases $C_{r}=1$. The radius has been normalized by the radius of maximum winds and the azimuthal wind has been normalized by its maximum value.

balance radiative subsidence in the outer region, this entails too shallow a profile of azimuthal velocity there.

As a test of whether the simple intensification model presented here captures the essential ingredients of storm amplification, we compare these results to simulations performed using the nonhydrostatic, axisymmetric model of RE87, modified to ensure that the difference equations conserve energy. We use the same parameter settings as described in Part I, but to facilitate comparison with the simple model we omit the pressure dependence of the saturation specific humidity of the sea surface, omit dissipative heating, and omit the wind dependence of the surface exchange coefficients. The vertical and horizontal mixing lengths in these simulations are both set to $1000 \mathrm{~m}$ (as in Part I); these are large enough to prevent the boundary layer flow from becoming appreciably supergradient (Persing and Montgomery 2003; Bryan and Rotunno 2009).

The model developed here assumes that the initial vortex is already well developed, in the sense that the core is already saturated with water vapor and a welldeveloped anticyclone exists near the tropopause. By contrast, the vortex used to initialize simulations using the RE87 model is not saturated anywhere and has no anticyclone at the tropopause. For these reasons, the starting vortex initially decays with time, until Ekman pumping has sufficiently moistened the inner core of the disturbance, at which time intensification begins. In these simulations, we subjectively determined an "ignition time" at which real intensification commences.

Figure 3 compares (19) with subjectively determined ignition times to the time evolution of maximum wind speed in the RE87 simulations. Figure 3a shows the results of varying the drag coefficient while holding the enthalpy exchange coefficient constant, while in Fig. 3b, the drag coefficient is held fixed while the enthalpy exchange coefficient varies. In calculating the evolution from (19), the boundary layer depth was chosen to yield a good fit to the single case $C_{D}=C_{k}=1 \times 10^{-3}$ but held at that value for all the other cases. Note that changing the boundary layer depth is equivalent, through (12), to changing the values of $C_{k}$ and $C_{D}$ simultaneously. The maximum wind speed used in (19) was calculated using (18). Agreement is reasonably good, except in the case $C_{D}=4 \times 10^{-3}, C_{k}=2 \times 10^{-3}$, in which case the RE87
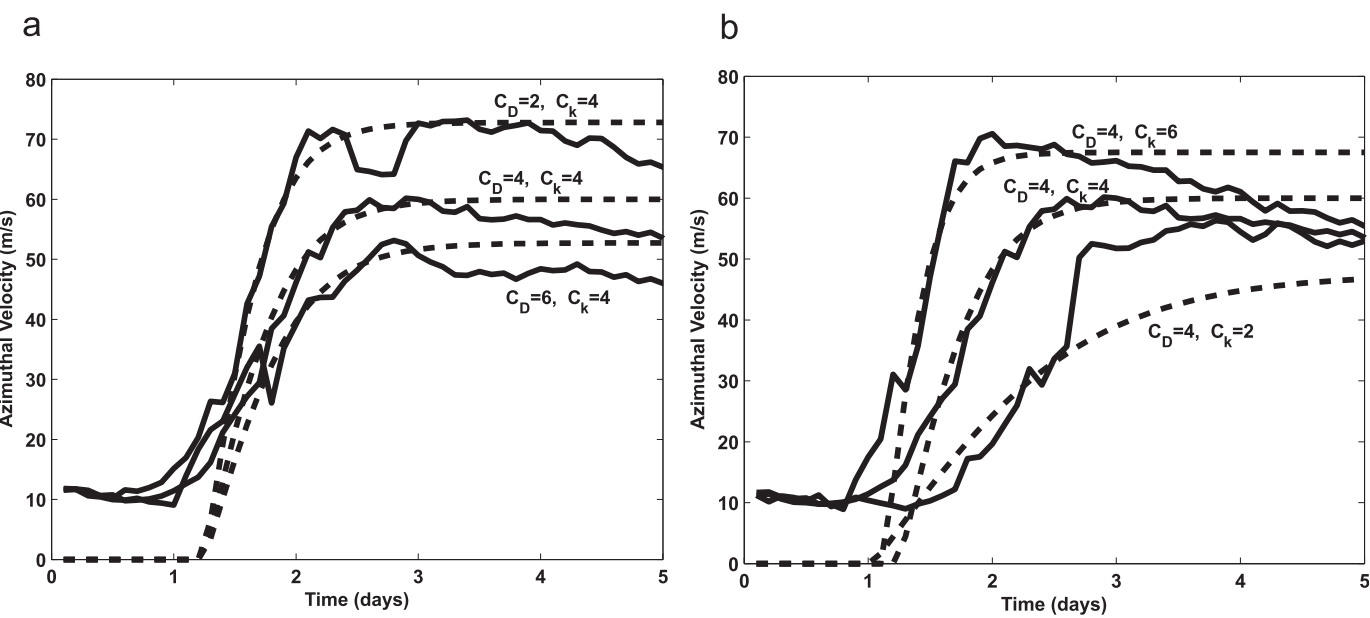

FIG. 3. Evolution with time of the domain maximum azimuthal wind in simulations using the RE87 model (solid), compared with solutions of (19) (dashed), varying (a) the drag coefficient and (b) the enthalpy exchange coefficient. Curves are labeled with the values of the exchange coefficients in units of $10^{-3}$. 
model reaches a higher intensity than predicted and does so somewhat more rapidly during the last phase of intensification.

\section{Discussion and summary}

While there is compelling evidence that tropical cyclones intensify and are maintained by surface enthalpy fluxes, previous work demonstrated that the spatial relationship between surface enthalpy fluxes and saturation entropy does not conduce to intensification. E97 showed that this problem can be alleviated by invoking a radial gradient of turbulent entropy fluxes out of the boundary layer in the region near the eyewall, but it is not clear from models or observations whether the required gradients actually exist. Moreover, it would be difficult to argue that such fluxes play any important role in the evolution of dry, surface flux-driven vortices as have been simulated recently by Mrowiec et al. (2011). Here we showed that even in the absence of radial gradients of turbulent entropy fluxes out of the boundary layer, tropical cyclones can intensify owing to gradients of outflow temperature across the eyewall. In Part I of this pair of papers, we showed that in numerical simulations of tropical cyclones, such outflow temperature gradients do exist and are consistent with the hypothesis that outflow thermal stratification is determined by small-scale turbulence that acts to prevent the $\mathrm{Ri}$ chardson number from falling below a critical value. We also demonstrated that this hypothesis leads to predictions of storm intensity and structure that are in good agreement with numerical simulations. Here we show that, in addition, the critical Richardson number hypothesis leads to predictions of storm evolution that are also, for the most part, in good accord with numerical simulations.

The basic physics at work here have to do with the spatial relationship between surface fluxes of entropy, which are partially controlled by surface wind speed, and the existing gradient of entropy. The thermal wind relation for a well-developed vortex, given by (2), shows that the surface wind speed is proportional to the gradient of entropy with respect to angular momentum multiplied by $T_{s}-T_{o}$ and weighted by physical radius. But for the entropy gradient to amplify, the entropy flux from the surface must itself have a radial gradient that is correlated with the existing radial entropy gradient. (The amplification of the radial entropy gradient, corresponding through the thermal wind relation to amplification of the azimuthal wind, may also be interpreted as an increase in the available potential energy of the system.) Thus, for the storm to amplify, the surface entropy flux must have a radially inward gradient across the radius of maximum winds. The entropy flux is proportional to the product of the surface wind speed and the air-sea entropy difference. The latter generally increases with radius and so works in the wrong direction in weighting the entropy fluxes toward smaller radii. If $T_{o}$ were constant, then according to (2) the surface wind speed would be a maximum outside the radius where the entropy gradient has its maximum (negative) value, and thus the radial gradient of surface fluxes across the radius of maximum wind would be of the opposite sign as the existing entropy gradient and the vortex would decay.

In E97 it was proposed that the missing ingredient is turbulent fluxes of entropy out of the boundary layer. If there is a sufficiently strong gradient of these across the radius of maximum winds, then the radial gradient of net entropy tendency can be negative at the radius of maximum winds, leading to an amplification of the entropy gradient and thus of the maximum wind itself. E97 also showed that a positive gradient in thermodynamic boundary layer depth across the radius of maximum winds could lead to intensification of the system by accelerating the increase in boundary layer entropy at small radii.

In the present paper, we neglect any radial gradient of boundary layer depth or turbulent entropy flux out of the boundary layer and focus on the factor $T_{s}-T_{o}$ that appears in (2) (and which was held constant in E97). This is essentially the integration constant that appears in integrating the thermal wind relation down from the temperature $T_{o}$ at which the azimuthal wind vanishes (by definition). If this outflow temperature increases across the radius of maximum winds $r_{m}$ (as they should, by definition), as is true here, then by (2) the surface winds decrease across $r_{m}$ (as they should, by definition), causing the required negative gradient in surface entropy flux so that the magnitude of the gradient can amplify. Thus, a negative gradient of the outflow temperature across the angular momentum surface corresponding to the radius of maximum winds, as observed in the numerical simulations reported in Part I, can allow a vortex to amplify even in the absence of radial gradients of turbulence entropy fluxes out of the boundary layer.

If the findings of Part I and the present paper withstand scientific scrutiny, it will be a remarkable fact that major properties of tropical cyclones, including their intensity, structure, and temporal evolution, all depend on small-scale turbulence in the outflow regions of the storms, which acts to set the thermal stratification of the outflow.

Acknowledgments. The author was supported by the National Science Foundation under Grant 0850639. 


\section{REFERENCES}

Bister, M., and K. A. Emanuel, 1998: Dissipative heating and hurricane intensity. Meteor. Atmos. Phys., 65, 233-240.

Bryan, G. H., and R. Rotunno, 2009: Evaluation of an analytical model for the maximum intensity of tropical cyclones. J. Atmos. Sci., 66, 3042-3060.

Emanuel, K. A., 1986: An air-sea interaction theory for tropical cyclones. Part I. J. Atmos. Sci., 43, 585-605.

, 1997: Some aspects of hurricane inner-core dynamics and energetics. J. Atmos. Sci., 54, 1014-1026.

_ 2007: Environmental factors affecting tropical cyclone power dissipation. J. Climate, 20, 5497-5509.

—_, and R. Rotunno, 2011: Self-stratification of tropical cyclone outflow. Part I: Implications for storm structure. J. Atmos. Sci., 68, 2236-2249.
Hakim, G. J., 2011: The mean state of axisymmetric hurricanes in statistical equilibrium. J. Atmos. Sci., 68, 1364-1376.

Mrowiec, A. A., S. T. Garner, and O. M. Pauluis, 2011: Axisymmetric hurricane in a dry atmosphere: Theoretical framework and numerical experiments. J. Atmos. Sci., 68, 1607-1619.

Persing, J., and M. T. Montgomery, 2003: Hurricane superintensity. J. Atmos. Sci., 60, 2349-2371.

Riehl, H., 1950: A model for hurricane formation. J. Appl. Phys., 21, 917-925.

Rotunno, R., and K. A. Emanuel, 1987: An air-sea interaction theory for tropical cyclones. Part II: Evolutionary study using a nonhydrostatic axisymmetric numerical model. J. Atmos. Sci., 44, $542-561$.

Zhang, J. A., R. F. Rogers, D.S. Nolan, and F. D. Marks, 2011: On the characteristic height scales of the hurricane boundary layer. Mon. Wea. Rev., 139, 2523-2535. 